\title{
Multi-modality Therapy Leads to Longer Survival in Primary Central Nervous System Lymphoma Patients
}

\author{
Dhruv Singhal, Timothy F. Witham, Anand Germanwala, John C. Flickinger, \\ David Schiff, Douglas Kondziolka
}

\begin{abstract}
Background: Primary central nervous system lymphoma (PCL) is more frequently encountered by neurosurgeons given the increasing incidence among both nonimmunocompromised and immunocompromised patients. The most frequent surgery is stereotactic biopsy. Historically, radiation therapy has been the standard treatment modality for this disease and median survival was in the 15-month range. More recently, multi-modality therapy combining radiation therapy with chemotherapy (systemic, intrathecal, and/or intra-arterial) have resulted in longer survivals. We reviewed survival data for our series of patients treated for PCL over the last decade. Methods: Thirty-four patients with histologically confirmed PCL were treated at our center. Multivariate Cox regression analysis was performed to determine which factor(s) (age, gender, HIV status, Karnofsky Performance Scale, chemotherapy, single modality therapy, histology, location, number of lesions, surgical resection) had a significant impact on survival. Results: The overall median survival was 19 months. Patients receiving multi-modality therapy $(n=17)$ (chemotherapy and radiation) had a median survival of 34 months compared to four months for patients receiving single modality therapy ( $\mathrm{n}=17$ including seven HIV positive patients). Multi-modality therapy was the only significant factor affecting survival in this multivariate analysis ( $<<0.0001)$. Conclusions: Chemotherapy plus radiotherapy significantly enhances survival over patients treated with single modality therapy alone. Quality of life issues should be addressed on a case by case basis as additional treatment modalities are initiated.
\end{abstract}

RÉSUMÉ: La thérapie multimodale prolonge la survie des patients atteints de lymphome du SNC. Introduction: Le lymphome primitif du SNC est plus fréquemment rencontré par les neurochirurgiens à cause de son incidence croissante tant parmi les patients non immunocompromis que les patients immunocompromis. La chirurgie la plus courante est la biopsie stéréotaxique. Historiquement, la radiothérapie était le traitement standard de cette maladie et la survie médiane était de l'ordre de 15 mois. Récemment, la thérapie multimodale combinant la radiothérapie et la chimiothérapie (systémique, intrathécale et/ou intra-artérielle) a prolongé la survie. Nous revoyons les données sur la survie dans notre série de patients traités pour lymphome primitif du SNC pendant la dernière décennie. Méthodes: Trente-quatre patients porteurs d'un lymphome primitif du SNC confirmé en anatomopathologie ont été traités dans notre centre. L'analyse de régression multivariée de Cox a été utilisée pour déterminer quel(s) facteur(s) (l'âge, le genre, le statut VIH, le score à l'échelle de performance de Karnofsky (KPS), la chimiothérapie, la thérapie unimodale (XRT), l'histologie, le site, le nombre de lésions, la résection chirurgicale) avait (avaient) un impact significatif sur la survie. Résultats: La survie médiane était de 19 mois. Les patients ayant reçu la thérapie multimodale $(\mathrm{n}=17)$ (chimiothérapie et radiothérapie) avaient une survie médiane de 34 mois versus quatre mois pour les patients recevant XRT ( $\mathrm{n}=17$, incluant sept patients VIH positifs). La thérapie multimodale était le seul facteur significatif affectant la survie dans cette analyse multivariée $(\mathrm{p}<0,0001)$. Conclusions: La chimiothérapie associée à la radiothérapie augmente significativement la survie par rapport à la XRT seule. Les questions relatives à la qualité de vie devraient être considérées cas par cas lorsque des modalités de traitement additionnelles sont entreprises.

Can. J. Neurol. Sci. 2002; 29: 147-153

Primary central nervous system lymphoma (PCL) is an uncommon type of nonHodgkin's lymphoma (NHL) affecting the central nervous system (CNS); making up $2 \%$ of all NHL and accounting for $5 \%$ of primary brain tumors. ${ }^{1}$ Primary central nervous system lymphoma is most frequently encountered in immunocompromised individuals. Specifically, patients who are HIV infected or immunosuppressed due to organ transplantation are at an increased risk of PCL. ${ }^{1}$ The incidence of PCL is $2-10 \%$ in the AIDS population. ${ }^{2}$ Immunosuppressed patients are at a 300 times greater risk of getting PCL than immunocompetent individuals. Over the past two decades the incidence of PCL in immunocompetent individuals has increased. ${ }^{1}$ The increase in the incidence of PCL cannot be solely attributed to improved neurodiagnostic procedures because this increase has been significantly greater than the increase in all other brain tumors. ${ }^{3}$ One case-control risk factor study in immunocompetent patients

From the Departments of Neurological Surgery and Radiation Oncology and the Center For Image-Guided Neurosurgery, University of Pittsburgh, Pittsburgh, Pennsylvania, USA.

ReCEIVED SePtember 27, 2001. ACCEPTEd In Final FORM JANUARY 9, 2002. Reprint requests to: Douglas Kondziolka, Department of Neurological Surgery, University of Pittsburgh Medical Center, 200 Lothrop Street, Suite B-400, Pittsburgh, PA 15213 USA 
suggested decreased education as a risk factor and tonsillectomy or prior oral contraceptive use as protective factors. ${ }^{4}$

Previous conventional treatment for PCL has been whole brain radiation therapy in combination with corticosteroids. ${ }^{1}$ Median overall survival with this regimen ranges between 12-16 months, though it is only two to five months in immunosuppressed patients. ${ }^{1,5}$ In most series, 50 Gy provides the maximal treatment effect but can be associated with long-term cognitive decline. Doses greater than 50 Gy are associated with post-radiation encephalopathy in long term survivors. ${ }^{6}$ Primary central nervous system lymphoma survival is not correlated with the extent of surgical tumor resection. ${ }^{1}$ Surgical resection as a single treatment modality results in a median survival of 3.5-5 months. ${ }^{3}$ Surgical intervention usually consists of stereotactic biopsy. Multi-modality treatment combining radiation with chemotherapy is now a standard therapy for patients who can tolerate these approaches. Chemotherapy can be divided into two groups based on whether the agents do or do not cross the blood brain barrier (BBB). Examples of chemotherapeutics that cross the $\mathrm{BBB}$ are high dose methotrexate and ara-c. Drugs that do not penetrate the $\mathrm{BBB}$ include cyclophosphamide, doxorubicin, vincristine, and prednisone (CHOP) and its derivatives.

Since the mid-1990s, vigorous multi-modality therapy combining chemotherapy and radiation has been the preferred treatment protocol at the University of Pittsburgh. The only patients to receive radiation therapy alone for PCL at our institution were treated before 1994. We reviewed management outcomes in patients treated for PCL over the last decade to test the hypothesis that those patients that received multi-modality treatments combining radiation and chemotherapy would have better survival outcomes than those patients receiving radiation with corticosteroids.

\section{METHODS AND MATERIALS}

A retrospective review was performed on all of the patients that were treated for PCL at the University of Pittsburgh from May 1988 to June 1998 ( $\mathrm{n}=35)$. The study was limited to patients with a confirmed histological diagnosis of PCL. One patient was excluded from the study because the treatment protocol was unknown. Therefore, 34 patients were included and their medical records were reviewed. Variables examined included gender, age at diagnosis, initial Eastern Conference Oncology Group (ECOG) score, ${ }^{7}$ initial Karnofsky Performance Scale (KPS), number of lesions, site of the lesion(s), HIV status, method of diagnosis, histology of the lymphoma, method of treatment, and length of survival (LOS). Length of survival was measured from the date of diagnosis to the date of death. Six patients are alive and survival for them has been recorded to date.

Seventeen patients were treated with whole brain radiation therapy alone, with dosages varying between 2000 cGy (w/boost) and $5000 \mathrm{cGy}(\mathrm{w} / \mathrm{boost})$. One patient had a dose that was not known due to incomplete records.

Seventeen patients were treated with a combination of radiation and chemotherapy. All patients except one received whole brain radiation with doses varying between 2800 - 5040 cGy. One patient received gamma knife radiosurgery to a maximum dosage of 3200 cGy. Seven patients received BBB penetrant chemotherapy including a combination of intrathecal methotrexate, high dose systemic methotrexate, or ara-c. Four patients received non-BBB penetrant chemotherapeutics consisting of systemic CHOP. Six patients received a combination of non-BBB and BBB penetrant chemotherapy.

A multivariate statistical analysis was performed on the data using the Cox proportional hazard analysis to determine which factors (gender, age at diagnosis, ECOG score, KPS, number of lesions, site of the lesion(s), HIV status, method of diagnosis, histology of the lymphoma, method of treatment) significantly affected the LOS. Since treatment began almost immediately after diagnosis, the survivals from date of diagnosis or treatment are considered to be the same.

\section{Results}

A summary of the patient demographics is shown in Table 1 . Twenty-four of the patients were male and 10 were female. The average age of the patients at diagnosis was 60.5 years (range 2385 years, median 64.5 years). The average ECOG score was 2.4 (range 1-4, median 2). The average KPS score at the time of diagnosis was 61.1 (range 30-80, median 60). The average number of lesions noted per patient was 1.8 (range 1-6, median 1). The site most frequently affected was the frontal lobe (14 lesions). The other focal locations included the temporal lobe (9), parietal lobe (7), basal ganglia (4), corpus collosum (5), brainstem (5), thalamus (2), interhemispheric fissure (1), ventricle (1), globus pallidus (1), optic nerve (1), and cerebellum (1). Twenty-seven patients were HIV negative and seven tested positive. Twenty-four patients were diagnosed by surgical biopsy, eight were diagnosed by stereotactic biopsy, and two were diagnosed by gross total resection (GTR). The most frequent histological finding was diffuse large cell with B-cell phenotype (91\%). Three patients $(9 \%)$ presented with the T-cell phenotype.

The average age of patients $(n=17)$ receiving single modality therapy alone was 59 years (median 64 years) and their average ECOG and KPS scores were 2.6 (median 2) and 55 (median 60) respectively. The average number of lesions for patients in this group was 2.3 (median 2$)$. The average age of patients $(n=17)$ receiving multi-modality therapy was 63 years (median 65 years) and their average ECOG and KPS scores were 1.9 (median 2) and 68 (median 70) respectively. The average number of lesions for patients in this group was 1.4 (median 1). The Cox proportional hazard analysis, which included variables for gender, age at diagnosis, ECOG score, KPS, number of lesions, HIV status, method of diagnosis, histology of the lymphoma, and method of treatment, indicated that the multi-modality treatment was the only significant factor having a positive impact on survival $(\mathrm{p}<0.0001)$.

The median LOS of the patients receiving multi-modality treatment was 34.0 months (range 8.5 - 108.0 months). The median LOS of the 17 patients who underwent single modality therapy was 4.5 months (range $0.5-26.0$ months). All the patients who were HIV positive $(n=7)$ received single modality therapy. Median survival of these patients was 4.0 months (range .75-26.0 months). The median survival of the remaining immunocompetent patients in the single modality group $(n=10)$ was 5.6 months (range 0.5 - 25.0 months).

Data of radiographic response to treatment were attainable for 
A
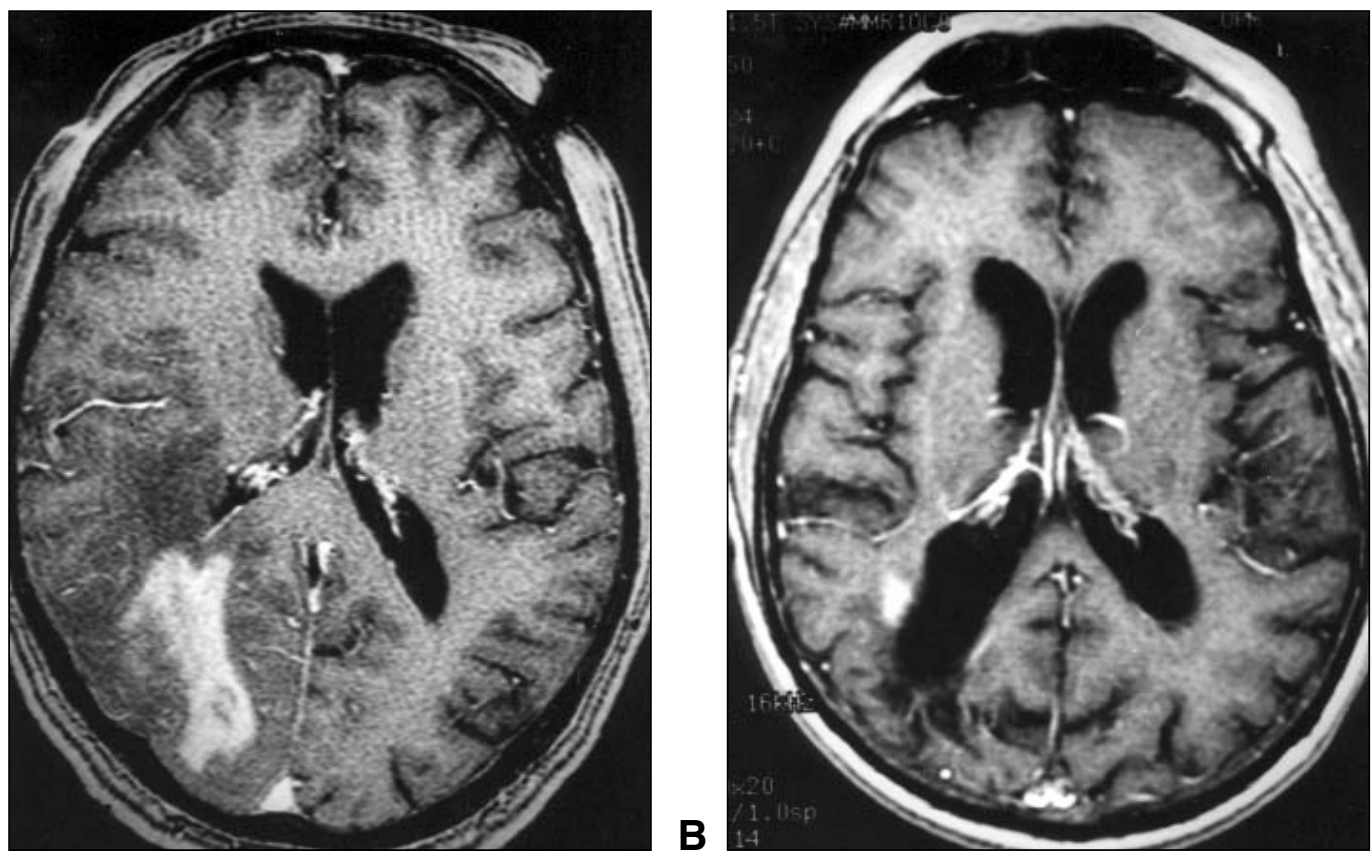

Figure 1: (A) A pre-treatment T1-weighted axial magnetic resonance image with contrast of patient \#33 showing a single lymphoma in the parietal lobe. (B) A post-treatment magnetic resonance image taken one year after diagnosis showing regression of parietal lobe lymphoma following multi-modality regimen consisting of high dose multi-modality treatment with ara-c and vincristine followed by leukovorin and whole brain radiation.
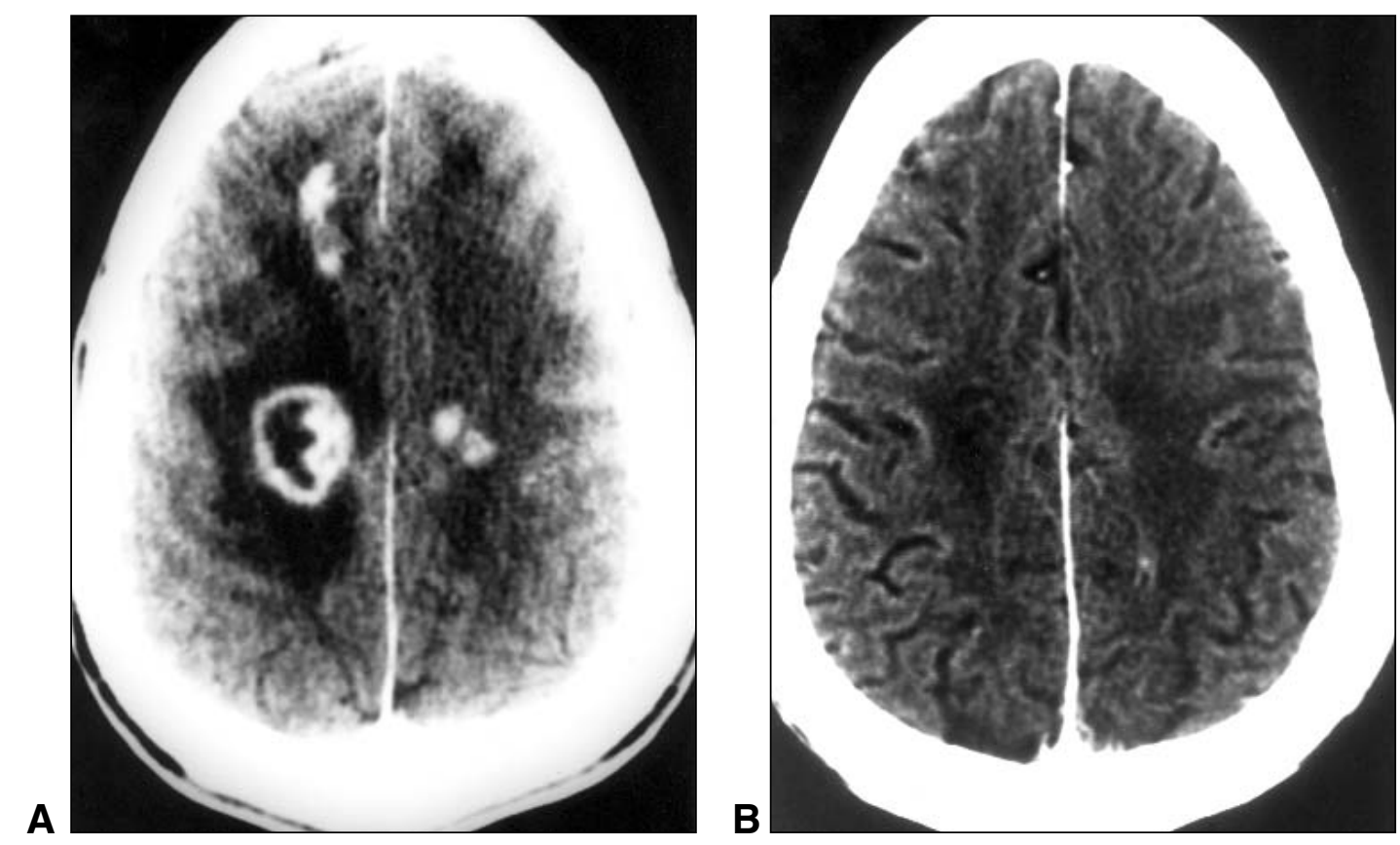

Figure 2: (A) A pre-treatment computed tomography (CT) scan with contrast of patient \#28, a HIV positive patient with multiple frontal lobe lymphomas and a right basal ganglia lymphoma. Ring-enhancing lesions, normally atypical for PCL, suggest the presence of HIV. (B) A post-treatment CT scan taken two months later following single modality treatment consisting of whole brain radiation showing complete resolution of frontal lobe and basal ganglia lymphomas. 
Table 1: Patient Demographics

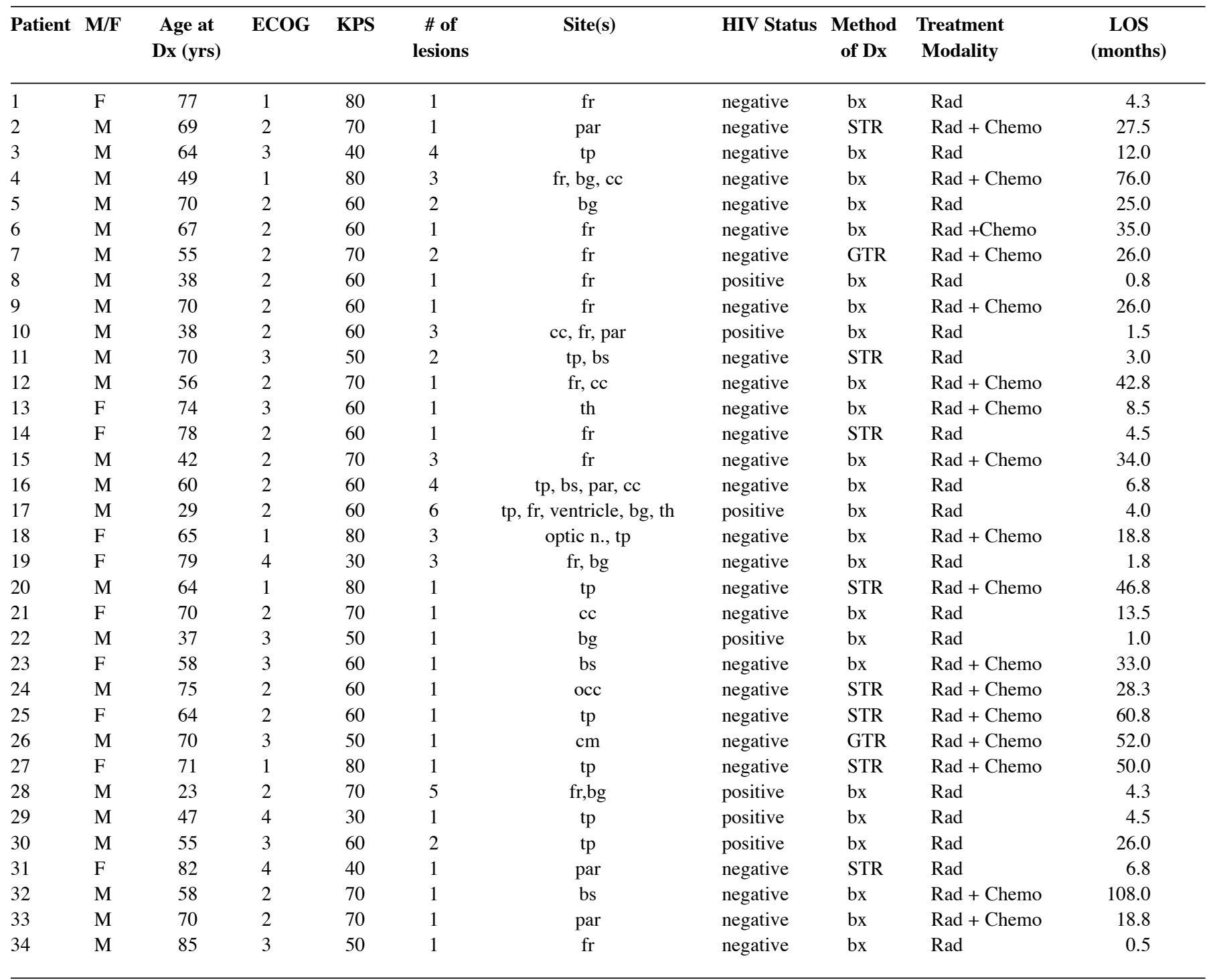

$\mathrm{cc}=$ corpus callosum, $\mathrm{bg}=$ basal ganglia, $\mathrm{tp}=$ temporal, $\mathrm{fr}=$ frontal, $\mathrm{par}=$ parietal, $\mathrm{cm}=$ cerebellum, occ $=$ occipital, $\mathrm{bs}=\mathrm{brainstem}, \mathrm{th}=\mathrm{thalamus}$ ECOG $=$ Eastern Conference Oncology Group score, KPS = Karnofsky Performance Scale, LOS = length of survival, Rad = radiation, Chemo = chemotherapy, GTR = gross total resection

nine patients who received multi-modality treatment (Figure 1). Follow-up varied from five to 35 months after the initiation of treatment (Figures $2 \& 3$ ). Five of the nine patients showed early responses to treatment but ultimately had radiographic evidence of progressive tumor growth. Three patients showed early responses and maintained a complete response throughout follow-up. Patient \#32 showed an early complete response but relapsed two years after treatment initiation. After receiving radiosurgery, this patient showed a complete radiographic response nine months later (Figure 4). Two patients presented with new tumors during follow-up (Table 2).

In eight patients with detailed follow-up for their entire clinical course, toxicity data related to tumor management were available. These included three patients with leukoencephalopathy, one with mild peripheral neuropathy related to vincristine, one with hydrocephalus and a chronic subdural hematoma, and one with a nocardia brain abscess.

\section{Discussion}

The survival benefit of chemotherapy in combination with radiation therapy as a first-line treatment for patients with PCL is well-documented. ${ }^{8-11}$ O'Brien et $\mathrm{al}^{11}$ reported a 33-month median survival for PCL patients receiving intravenous methotrexate prior to radiation. DeAngelis et $\mathrm{al}^{5}$ reported a 41 month median recurrence time for immunocompetent patients 

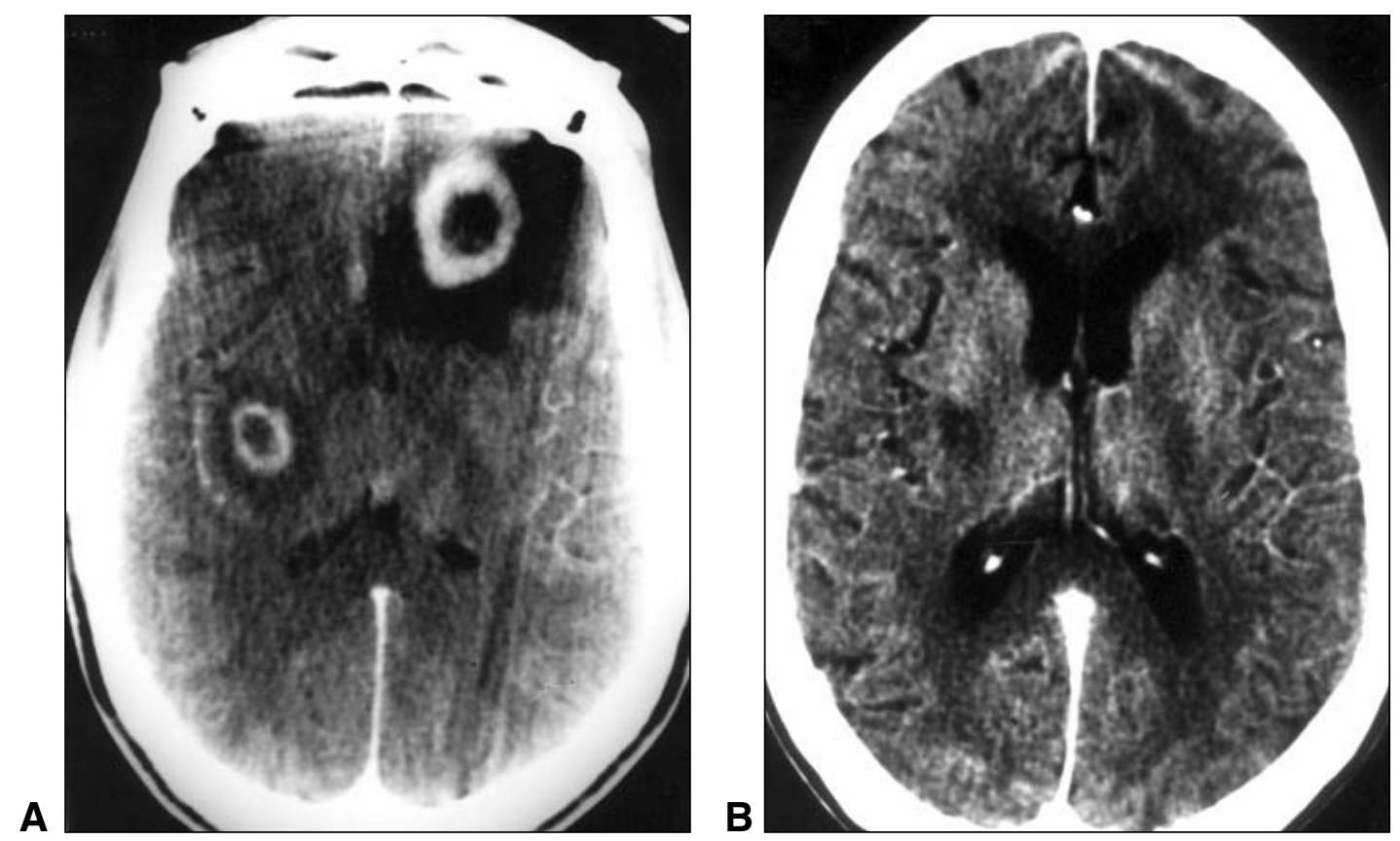

Figure 3: (A) A pretreatment CT scan with contrast of patient \#28 from a more caudal view than in Figure 2. (B) A posttreatment scan two months following whole brain radiation showing complete resolution.

A

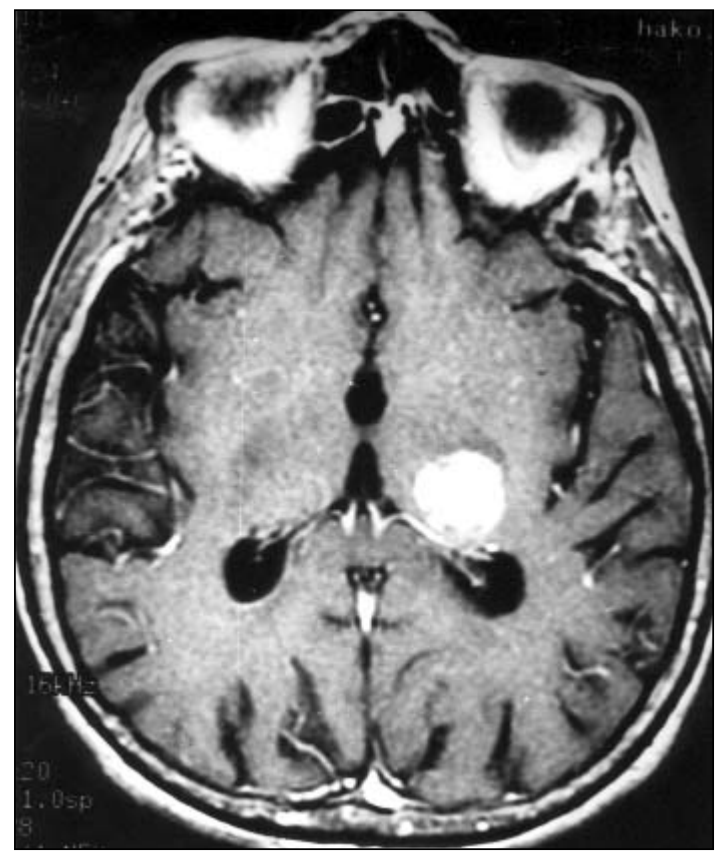

B

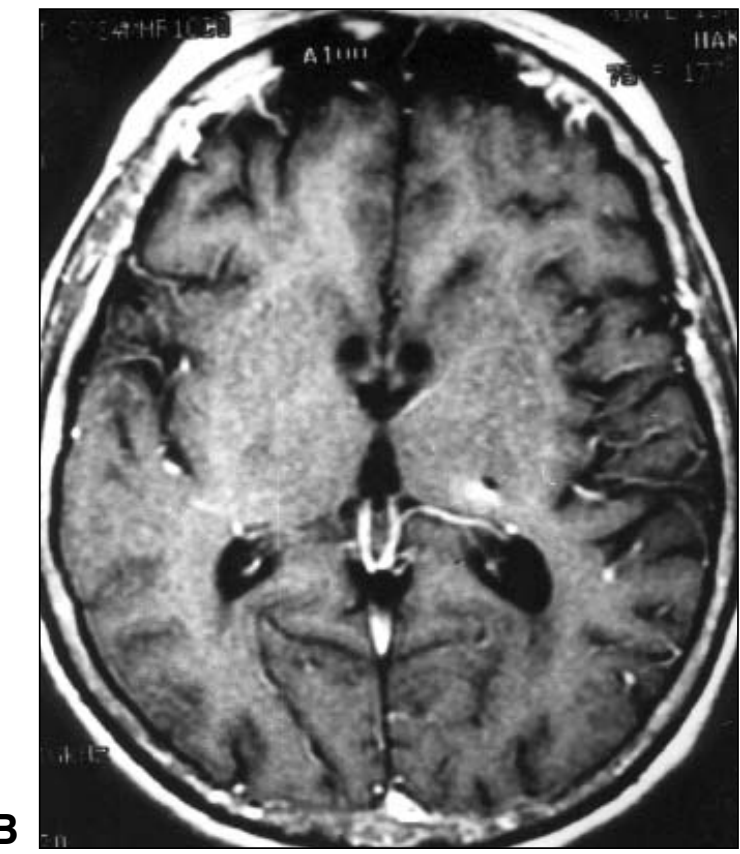

Figure 4: (A) A pre-treatment T1-weighted axial magnetic resonance image with contrast of patient \#13 showing a lymphoma of the left thalamus. (B) A post-treatment scan taken three months after diagnosis following multi-modality therapy consisting of high dose multi-modality treatment with leukovorin rescue and gamma knife radiosurgery. 
Table 2: Radiographic Follow-up of Patients Receiving Multimodality Therapy

\begin{tabular}{|c|c|c|c|c|c|}
\hline \multirow[t]{2}{*}{ Patient } & \multirow{2}{*}{\begin{tabular}{|l|} 
Months \\
Following \\
Treatment \\
Initiation
\end{tabular}} & \multicolumn{4}{|c|}{ Tumor Response: } \\
\hline & & $\begin{array}{l}\text { Complete } \\
\text { Response }\end{array}$ & $\begin{array}{l}\text { Partial } \\
\text { Response* }\end{array}$ & $\begin{array}{l}\text { No } \\
\text { Change }\end{array}$ & Progression \\
\hline 2 & $\begin{array}{l}20 \\
21 \\
22 \\
23\end{array}$ & $\begin{array}{l}X \\
X\end{array}$ & $\mathrm{X}$ & & $\begin{array}{c}\text { new tumor } \\
\mathrm{X}\end{array}$ \\
\hline 6 & $\begin{array}{l}1 \\
5\end{array}$ & $\mathrm{X}$ & $\mathrm{X}$ & & \\
\hline 9 & $\begin{array}{r}5 \\
6 \\
7 \\
10 \\
19 \\
\end{array}$ & & $\begin{array}{l}X \\
X \\
X \\
X\end{array}$ & & $X$ \\
\hline 12 & $\begin{array}{r}2 \\
3 \\
6 \\
7 \\
8 \\
12 \\
15 \\
18 \\
29 \\
32 \\
35 \\
\end{array}$ & $\begin{array}{l}\mathrm{X} \\
\mathrm{X} \\
\mathrm{X}\end{array}$ & $\begin{array}{l}X \\
X \\
X \\
X \\
X\end{array}$ & & $\begin{array}{l}X \\
X \\
X\end{array}$ \\
\hline 13 & $\begin{array}{l}2 \\
4 \\
5 \\
\end{array}$ & $\mathrm{X}$ & $\begin{array}{l}X \\
X\end{array}$ & & \\
\hline 15 & $\begin{array}{r}3 \\
7 \\
9 \\
10 \\
\end{array}$ & $\begin{array}{l}X \\
X \\
X\end{array}$ & & & $\begin{array}{l}\text { new tumor } \\
\text { new tumor } \\
X\end{array}$ \\
\hline$\overline{26}$ & $\begin{array}{r}6 \\
9 \\
13 \\
22 \\
\end{array}$ & $\begin{array}{l}X \\
X \\
X \\
X \\
\end{array}$ & & & \\
\hline 32 & $\begin{array}{r}2 \\
24 \\
33 \\
\end{array}$ & $\begin{array}{l}\mathrm{X} \\
\mathrm{X} \\
\end{array}$ & & (gamma) & \\
\hline 33 & $\begin{array}{r}3 \\
11 \\
13 \\
15\end{array}$ & & $\begin{array}{l}X \\
X\end{array}$ & & $\begin{array}{l}X \\
X\end{array}$ \\
\hline
\end{tabular}

*Partial response is a $25-50 \%$ reduction in tumor size

receiving systemic methotrexate prior to radiation followed by high dose ara-c treatment. There are no randomized comparisons of these two therapeutic approaches.

We reviewed our series of 34 patients with PCL whose median age of 64.5 years at diagnosis is similar to those reported in previous studies. ${ }^{1}$ The median age recorded in previous studies of HIV positive PCL patients at diagnosis is under 40 years and the age of HIV patients at diagnosis in our study was 38 years. ${ }^{1}$ In our series, $91 \%$ of the patients had B-cell lymphomas which supports the high prevalence of B-cell histology in other series. ${ }^{6}$ Forty-four percent of our patients presented with multi-focal lesions which have been reported to occur in at least $30 \%$ of the PCL population. ${ }^{1}$

Although the diagnosis of PCL can be suggested by neuroimaging, a diagnosis of PCL necessitates histological confirmation. Magnetic resonance (MR) imaging with gadolinium enhancement is the most sensitive test for PCL. ${ }^{6}$ Gadolinium studies show regions of BBB compromise which occurs near cerebral lymphomas. In immunocompetent individuals, certain characteristics of PCL can be recognized from a computed tomography (CT) or MR scan. Specifically, the mass will be diffusely enhancing with ill-defined borders and a variable amount of edema surrounding the mass. Diagnosis can be made confidently by demonstration of clearly malignant lymphocytes from cerebrospinal fluid or from uveal aspirate. The diagnosis is confirmed by surgical biopsy. A potential problem with obtaining a definitive biopsy exists if corticosteroids are given before a diagnosis has been confirmed. Steroids, which are given to reduce swelling, also cause tumor cell lysis in PCL. The tumor may disappear, leading to a nondiagnostic biopsy, only to relapse again. ${ }^{1,12}$ Staging is accomplished with cerebrospinal fluid analysis and ophthalmologic exam. ${ }^{12}$ In patients with AIDS, the diagnosis may not be as straightforward. Primary central nervous system lymphoma often exhibits ring enhancement, which occurs from central necrosis, and therefore makes lymphomas appear identical to other lesions such as toxoplasmosis. ${ }^{13,14}$ Primary central nervous system lymphoma in an AIDS patient may be suggested through single photon emission computed tomography and a positive finding of Epstein-Barr virus (EBV) DNA in the cerebral spinal fluid. $^{12}$

The etiology of PCL in immunocompromised individuals is better understood than its origins in the immunocompetent population. The EBV infects B-lymphocytes and is latent in these cells. When an individual is immunocompromised, the suppressor T-cells that control the EBV-infected B-lymphocytes are not present and the B-lymphocytes proliferate into a tumor. The tumor is thought to occur in the CNS because of minimal cerebral immune surveillance. In immunocompetent individuals, the etiology is not as clear. Although T-cells normally travel into the CNS, the same is not true for B-cells and yet $90 \%$ of PCL cases are diffuse large B-cell lymphomas. ${ }^{1}$ DeAngelis ${ }^{12}$ offers three distinct theories for B-cell penetration into the CNS. One theory is that a systemic B-cell lymphoma settles into the CNS. Another potential explanation is the transition and growth of lymphocytes that normally traffic the CNS. Finally, an "antigenic stimulus" may attract the B-cells to the CNS.

The use of both BBB penetrant and nonpenetrant chemotherapeutic agents with radiation for the treatment of PCL have been documented in the literature. Blay et $\mathrm{al}^{8}$ reports improved survival in patients receiving high dose methotrexate before radiation compared to other treatment regimens including whole brain radiation alone. Others report no significant effect of cyclophosphamide, doxorubicin, vincristine, and dexamethasone (CHOD) administered before radiation on survival compared to 
radiation alone. Reviews of the literature report a greater sensitivity of PCL to BBB-penetrant chemotherapeutic agents, particularly high dose methotrexate, in comparison to the nonBBB penetrant CHOP derivatives. ${ }^{5,6}$

The review of our data reveals a significant difference $(\mathrm{p}<0.0001)$ in LOS between those patients receiving chemotherapy and radiation versus whole brain radiation alone. The median survival from the single modality treatment $(n=17)$ was 4.5 months versus 34 months for multi-modality regimens $(n=17)$. All of the HIV patients from our review received radiation therapy alone. Despite previous reports ${ }^{5}$ that AIDS patients have lower survival outcomes from whole brain radiation than immunocompetent patients, we found that the HIV patients did not exhibit a significantly lower survival $(n=7$, median 4.0 months, range $.75-26.0$ months) than the immunocompetent patients $(n=10$, median 5.6 months, range 0.5 - 25.0 months) in the single modality group. Immunocompetent patients in our study receiving single modality treatment exhibited 7-13 months shorter survival than reported studies of other immunocompetent patient populations receiving single modality therapy. ${ }^{5}$ We speculate that the advanced age of our immunocompetent patients $(n=10$, median 70 years, average 73.5 years) relative to other similar studies predisposed our patients to additional co-morbidities that affected their survival after whole brain radiation.

As the management of these lesions becomes more complex it will be our obligation to prove that increased survival from the addition of new treatments results in acceptable quality of life. Therefore, we must assess the quality of life issues on a case-bycase basis as new modalities are initiated. As we learn more about the pathobiology of malignant brain tumors including PCL, it becomes apparent that a multi-modality approach to these lesions is necessary to enhance survival. It is clear that promising new modalities are on the horizon including immune modulation, gene therapy, and novel drug delivery techniques, among others. Our series, as have others, demonstrates that a multi-modality approach including chemotherapy and radiation therapy should be provided to patients with PCL. Radiation therapy is responsible for the neurotoxicity that continues to impair the quality of life of PCL patients. We acknowledge that elderly patients or medically infirm patients may not tolerate chemotherapy. In others, radiosurgery may provide an effective local tumor response but we believe that it is not the best first line therapy. We use radiosurgery in patients for tumor recurrence if additional chemotherapy is not suitable. We hope our study will encourage investigation into novel radio- and chemotherapeutic approaches towards PCL that may lead to improved clinical outcomes.

\section{REFERENCES}

1. Blay JY, Ongolo-Zogo P, Sebban C, et al. Primary cerebral lymphomas: unsolved issues regarding first-line treatment, follow-up, late neurological toxicity and treatment relapses. Ann Oncol 2000; 11(Suppl 1):S39-S44.

2. Nasir S, DeAngelis LM. Update on the management of primary CNS lymphoma. Oncology 2000; 14(2):228-234, 237-242, 244.

3. Reni M, Ferreri AJM, Garancini MP, Villa E. Therapeutic management of primary central nervous system lymphoma in immunocompetent patients: results of a critical review of the literature. Ann Oncol 1997; 8:227-234.

4. Schiff D, Suman VJ, Yang P, Rocca WA, O’Neill BP. Risk factors for primary central nervous system lymphoma: a case-control study. Cancer 1998; 82(5):975-982.

5. DeAngelis LM. Current management of primary central nervous system lymphoma. Oncology 1995; 9:63-71.

6. Abrey LE. Primary central nervous system lymphoma. Neurologist $2000 ; 6: 245-254$.

7. Oken MM, Creech RH, Tormey DC, et al. Toxicity and response criteria of the Eastern Cooperative Oncology Group. Am J Clin Oncol 1982; 5:649-655.

8. Blay JY, Conroy T, Chevreau C, et al. High dose methotrexate for treatment of primary cerebral lymphomas - analysis of survival and late neurologic toxicity in a retrospective series. J Clin Oncol 1998; 16:864-870

9. DeAngelis LM, Yahalom J, Heinemann M-H, et al. Primary CNS lymphoma: combined treatment with chemotherapy and radiotherapy. Neurology 1990; 40:80-86.

10. DeAngelis LM, Yahalom J, Thaler HT, Kher U. Combined modality therapy for primary CNS lymphoma. J Clin Oncol 1992; 10:635643.

11. O'Brien P, Roos D, Pratt G, et al. Phase II multicenter study of brief single-agent methotrexate followed by irradiation in primary CNS lymphoma. J Clin Oncol 2000; 18(3):519-526.

12. DeAngelis LM. Primary central nervous system lymphoma. Curr Opin Neurology 1999; 12:687-691.

13. Forsyth PA, Yahalom J, DeAngelis LM. Combined modality therapy in the treatment of primary central nervous system lymphoma in AIDS. Neurology 1994; 44:1473-1478.

14. Forsyth PA, DeAngelis LM. Biology and management of AIDSassociated primary CNS lymphomas. Hematol Oncol Clin North Am 1996; 10: 1125 -1134. 